\title{
Comparative Effects of Three Kinds of Bariatric Surgery: A Randomized Case-Control Study in Obese Patients
}

\author{
Dan Luo • Qingling Yang • Li Zhou • Haibo Wang • Feng Li • \\ Hailong Ge $\cdot$ He Huang · Jian Liu · Yanjun Liu (D)
}

Received: July 28, 2019 / Published online: December 3, 2019

(C) The Author(s) 2019

\begin{abstract}
Introduction: Laparoscopic bariatric surgery is necessary for obese patients who cannot control body weight through daily diet and exercise, or other non-surgical ways. Three kinds of laparoscopic bariatric surgery, namely Roux-en-Y gastric bypass, sleeve gastrectomy, and sleeve gastrectomy with jejunal bypass, are available for weight loss in clinical practice, but their comparative effects are unclear. In this study,
\end{abstract}

Dan Luo and Qingling Yang are co-first authors.

Enhanced Digital Features To view enhanced digital features for this article go to https://doi.org/10.6084/ m9.figshare.9995507.

D. Luo $\cdot$ L. Zhou $\cdot$ H. Wang $\cdot$ F. Li $\cdot$ H. Ge .

H. Huang · J. Liu ( $₫)$

Department of General Surgery, Chengdu Fifth

People's Hospital, 33 Mashi St, Chengdu 610000,

Sichuan, China

e-mail: 501298916@qq.com

Q. Yang

Department of Respiratory, Chengdu Fifth People's Hospital, 33 Mashi St, Chengdu 610000, Sichuan, China

Y. Liu $(\bowtie)$

Department of General Surgery, The Third People's

Hospital of Chengdu, The Second Affiliated Chengdu Clinical College of Chongqing Medical

University, 82 Qinlong St, Chengdu 610031,

Sichuan, China

e-mail: liu103393828@126.com these were compared to illustrate their clinical effects.

Methods: A case-control study was conducted on 175 participants who fulfilled the inclusion criteria of laparoscopic bariatric surgery, while the controls were the same subjects before and after surgery, as well as with different surgeries specifically. Standardized weight loss measures were compared using analysis of covariance, with months from surgery as the covariant, including percentage total weight loss and excess body mass index (BMI) loss.

Results: A total of 175 patients were enrolled in this study (age $38 \pm 10$ years; BMI $46 \pm 5 \mathrm{~kg}$ / $\mathrm{m}^{2}$ ), with a mean postsurgery follow-up of $18 \pm 6$ months. No significant difference was included among the enlisted patients before bariatric surgery. However, after surgery according to personal health indexes of patients and professional assessment by doctors, patients specifically receiving one of Roux-en-Y gastric bypass, sleeve gastrectomy, and sleeve gastrectomy with jejunal bypass had no significant difference between weight loss and BMI, while the most important factors were dietary control and exercise after bariatric surgery.

Conclusion: This study suggests that bariatric surgery is only a prerequisite for weight loss, and the long-term dietary control and exercise can help patients achieve optimal weight loss. 
Keywords: Roux-en-Y gastric bypass; Sleeve gastrectomy; Sleeve gastrectomy with jejunal bypass

\section{Key Summary Points}

Why carry out this study?

Obesity has many negative effects on people's health, including obesity syndrome and complex complications and so on, causing heavy economic losses and social burdens.

When failing to rely on body management to lose weight, surgery is an effective alternative approach. Roux-en-Y gastric bypass (RYGB), sleeve gastrectomy, and sleeve gastrectomy with jejunal bypass (SGJB) are commonly adopted in clinical practice according to body index of patients; however, little is known about the difference of the three kinds of bariatric surgery.

So in order to provide specific and detailed illustration on the prognosis of the three kinds of bariatric surgery, we compared their clinical effects based on a case-control study of 175 participants.

What was learned from the study?

For overweight patients, it is hard to lose weight without bariatric surgery when failing to self-manage the condition.

There are no significant differences in the prerequisites for Roux-en-Y gastric bypass, sleeve gastrectomy, and sleeve gastrectomy with jejunal bypass, including age, sex, weight, BMI, and other medical comorbidities, except diabetes and hyperinsulinemia.

The three kinds of bariatric surgery have no significant difference between weight loss and BMI for a long time, while the most important factors were long-term dietary control and exercise after bariatric surgery for optimal weight loss.

\section{INTRODUCTION}

By reducing food intake and nutrition absorption, bariatric surgery is an effective treatment option for not only long-term weight loss [1] but also improving visceral fat and declining the risk of cardiovascular diseases [2], significantly ameliorating obesity-related comorbidities. The most common bariatric surgeries for obesity therapy are Roux-en-Y gastric bypass (RYGB) [3], sleeve gastrectomy [4], and sleeve gastrectomy with jejunal bypass (SGJB) [5]. RYGB surgery could reduce food intake and absorption by directly linking small stomach capsule and small intestine, which has been studied earlier and performed more frequently in patients with weight loss and diabetes. Moreover RYGB is most effective in treating patients with obesity accompanied by type 2 diabetes [6, 7]. Sleeve gastrectomy would remove about $70 \%$ of stomach volume and keep the main part responsible for digesting food. In addition to less intake, sleeve gastrectomy reduces the production of ghrelin to suppress appetite [8]. About 10-15 BMI points or $70 \%$ excess weight could decrease within 2 years after surgery. For those with a higher BMI (over 40) and difficulty in reducing their food intake, sleeve gastrectomy is ideal for weight loss, as is currently widely applied in Europe [9]. SGJB is an improvement of sleeve gastrectomy, specifically for the treatment of insulin resistance and type 2 diabetes $[10,11]$. However, little is known about differences of the three kinds of bariatric surgery. We performed a case-control study to survey 175 patients for 1-2 years undergoing RYGB, sleeve gastrectomy, or SGJB to evaluate and compare their clinical effects.

\section{METHODS}

\section{Participants}

All patients who participated in this study underwent bariatric surgery, including RYGB, sleeve gastrectomy, and SGJB, according to personal health conditions of patients and 
doctors' recommendations. Roux-en-Y gastric bypass is an end-to-side surgical anastomosis of the bowel involving creating a small pouch from the stomach and connecting the newly created pouch directly to the small intestine. Roux-en-Y gastric bypass is promising to improve the treatment of type 2 diabetes. Sleeve gastrectomy removes about $85 \%$ of the stomach leaving a cylindrical or sleeve shaped stomach with a capacity ranging from about 60 to $150 \mathrm{~cm}^{3}$, depending upon the surgeon performing the procedure. SGJB uses staples to create a sleeve of stomach. After this, a loop of the part of small intestine called the jejunum is joined to the first part of the duodenum. Weight and BMI were recorded before and after bariatric surgery. We comprehensively evaluated the health indexes of every patient and talked about the surgery with the patients for informed consent and permission, and eventually performed the operation.

The three kinds of bariatric surgery had the potential to cause blood clots, postoperative bleeding, infection, and pain. Blood clots were regularly cleaned up during the operation. Postoperative bleeding and pain were common in our patients, but they can be improved under proper hospital care. Infection was rare and only one patient was infected because of improper care after being discharged to his home, but after that he was quickly cured. In our hospital, every bariatric surgery was strictly evaluated and performed by experts, so the frequency of complications was very low. Once a syndrome was detected, doctors initiated treatment as soon as possible. We have done a baseline matching for any possible co-variates to rule out an inappropriate study population in advance. Patients were followed up by telephone interview once per month for 1-2 years to record data, including current body weight, daily diet, healthy status, and exercise habits.

\section{Compliance with Ethics Guidelines}

All patients have signed informed consent and agreed to the telephone interview as approved by our review board. This study protocol was approved our institutional ethical committee.
This study was performed in accordance with the Helsinki Declaration of 1964 and its later amendments.

\section{Physical Activity Measurement and Diet Management}

On the basis of the Behavioral Risk Factor Surveillance System Survey, we interviewed participants once per month by telephone [12]. Participants were asked questions about current weight, daily diet, and leisure-time exercise.

\section{Study Selection}

We performed a case-control study and metaanalysis in patients with obesity treated with bariatric surgery and accompanied by medical tests before and after surgery. Patients were acting as their own control group, since various physical signs were compared before (perioperative) and after the surgery. Patients undergoing reoperation for obesity were excluded.

\section{Data Extraction}

Data was extracted independently using standard data extraction forms, including weight, BMI, type 2 diabetes, hyperinsulinemia, hypertension, hyperuricemia, hyperlipidemia, nonalcoholic fatty liver disease, obstructive sleep apnea syndrome, sexual dysfunction, and liver function indexes, as well as the corresponding operation method. Disagreements were discussed and resolved by consultation between all authors.

\section{Risk of Bias}

Two reviewers independently evaluated the quality of the selected studies using the Newcastle-Ottawa scale (NOS) [13]. According to NOS, evaluation criteria were based on three methodological categories: selection (score 0-4), comparability (score 0-2), and outcome (score 0-3). Score 7-9 was considered high, 4-6 intermediate, and 0-3 low. Disagreements were excluded by consensus. 


\section{Statistical Analysis}

Meta-analysis was applied and the effects of the specific bariatric surgery were expressed with 95\% confidence intervals for dichotomous outcomes and mean difference (MD) for continuous outcomes with 95\% confidence intervals [14]. ANCOVA was used to remove the bias of other variables which may confound dependent variables. All statistical analyses were performed with SPSS 22.0, and $P<0.05$ was considered significant.

\section{RESULTS}

About $54 \%$ of the eligible patients were successfully enrolled; they completed tests every 6 months for 1-2 years to evaluate the effects of bariatric surgery. Among the 175 enrolled patients, RYGB surgery was adopted by 62 patients, sleeve gastrectomy by 78 patients, and SGJB by 35 patients. The average follow-up from the time of surgery was 1.8 years, with a range between 1 and 2 years (Fig. 1).
Presurgical characteristics of patients can be found in Table 1. Weight, BMI, and medical comorbidities were the most important factors when considering which kind of bariatric surgery was adopted. Moreover, we comprehensively evaluated the health indexes of every patient and talked about the surgery with patients for informed consent and permission, and eventually performed the operation. It is noteworthy that no matter what surgery type was adopted, there were no significant differences between the three groups with respect to age, sex, weight, BMI, and other medical comorbidities except diabetes and hyperinsulinemia; in relation to the surgery type, the percentage of patients who underwent sleeve gastrectomy was the lowest in those with diabetes and the highest in those with hyperinsulinemia. Moreover, the highest percentage of obese patients with diabetes underwent SGJB to achieve weight loss.

The subsequent weight tests after the corresponding bariatric surgery showed a time-dependent decrease in average percentage weight loss (Fig. 2). Comparatively, there were no

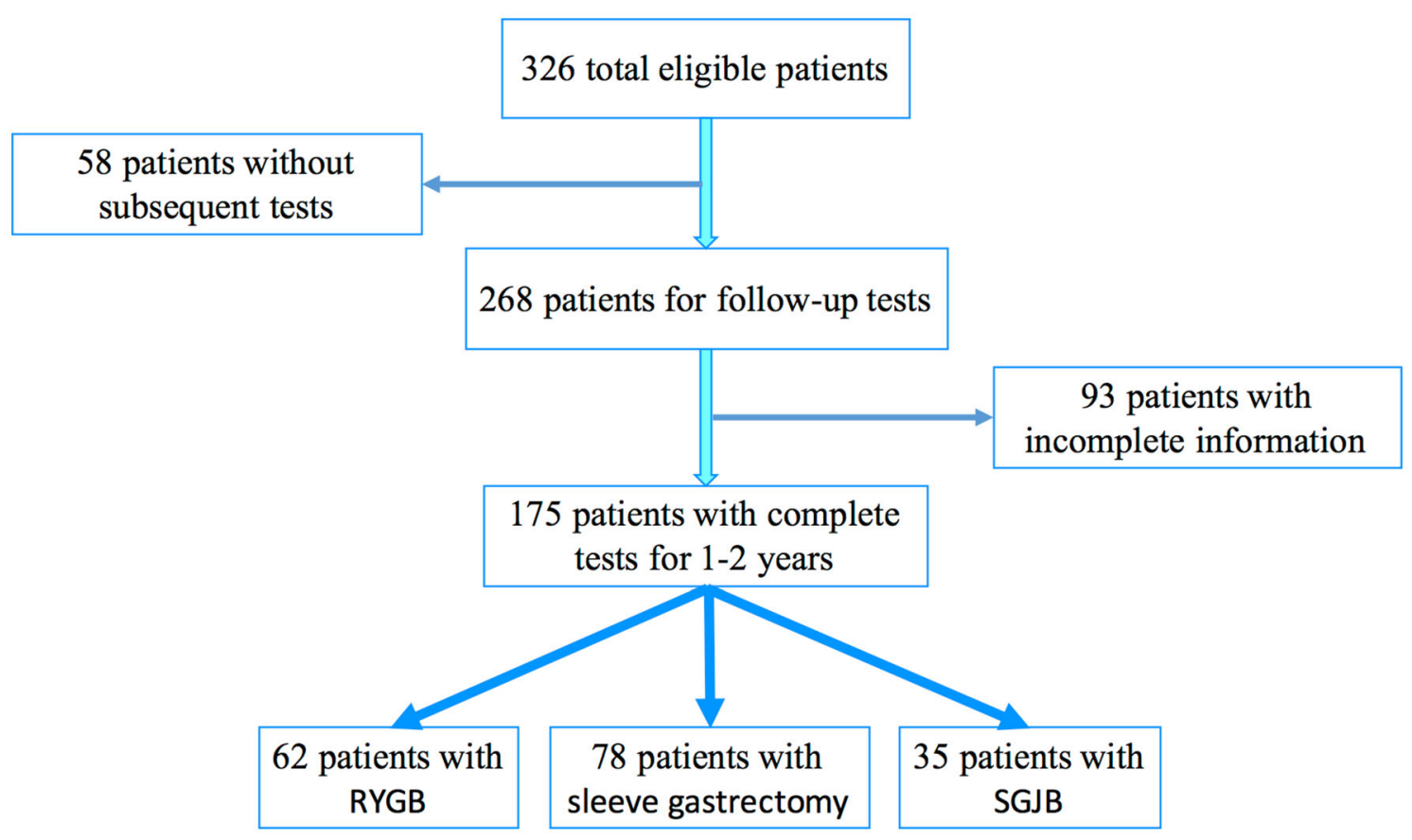

Fig. 1 Schematic diagram of enlisted patients and treatment 
Table 1 Descriptive baseline characteristics based on bariatric surgery

\begin{tabular}{llllll}
\hline Variable & Overall & RYGB & Sleeve gastrectomy & SGJB & $\boldsymbol{P}$ value \\
\hline Total $(N)$ & 175 & 62 & 78 & 35 & \\
Age $($ years $)$ & $38 \pm 10$ & $39 \pm 10$ & $37 \pm 12$ & $36 \pm 11$ & 0.412 \\
Female & $112(64 \%)$ & $41(66 \%)$ & $48(62 \%)$ & $23(66 \%)$ & 0.215 \\
Weight $(\mathrm{kg})$ & $138 \pm 16$ & $136 \pm 18$ & $142 \pm 16$ & $139 \pm 16$ & 0.132 \\
BMI $\left(\mathrm{kg} / \mathrm{m}^{2}\right)$ & $46 \pm 5$ & $47 \pm 5$ & $46 \pm 5$ & $46 \pm 6$ & 0.327 \\
Medical comorbidities & & & & & \\
$\quad$ Diabetes & $58(33 \%)$ & $24(39 \%)$ & $13(17 \%)$ & $21(60 \%)$ & 0.021 \\
Hyperinsulinemia & $62(35 \%)$ & $18(29 \%)$ & $35(45 \%)$ & $9(26 \%)$ & 0.031 \\
Hypertension & $74(42 \%)$ & $27(44 \%)$ & $31(40 \%)$ & $16(46 \%)$ & 0.127 \\
Hyperuricemia & $28(16 \%)$ & $10(16 \%)$ & $13(17 \%)$ & $5(14 \%)$ & 0.246 \\
Hyperlipidemia & $52(30 \%)$ & $17(27 \%)$ & $26(33 \%)$ & $9(26 \%)$ & 0.123 \\
NAFLD & $168(96 \%)$ & $60(97 \%)$ & $74(95 \%)$ & $34(97 \%)$ & 0.326 \\
OSAS & $72(41 \%)$ & $25(40 \%)$ & $32(41 \%)$ & $15(43 \%)$ & 0.512 \\
Sexual dysfunction & $4(2 \%)$ & $1(2 \%)$ & $2(2 \%)$ & $1(3 \%)$ & 0.102 \\
Liver function dysfunction & $36(21 \%)$ & $11(18 \%)$ & $16(21 \%)$ & $8(23 \%)$ & 0.135 \\
\hline
\end{tabular}

$N A F L D$ non-alcoholic fatty liver disease, $O S A S$ obstructive sleep apnea syndrome

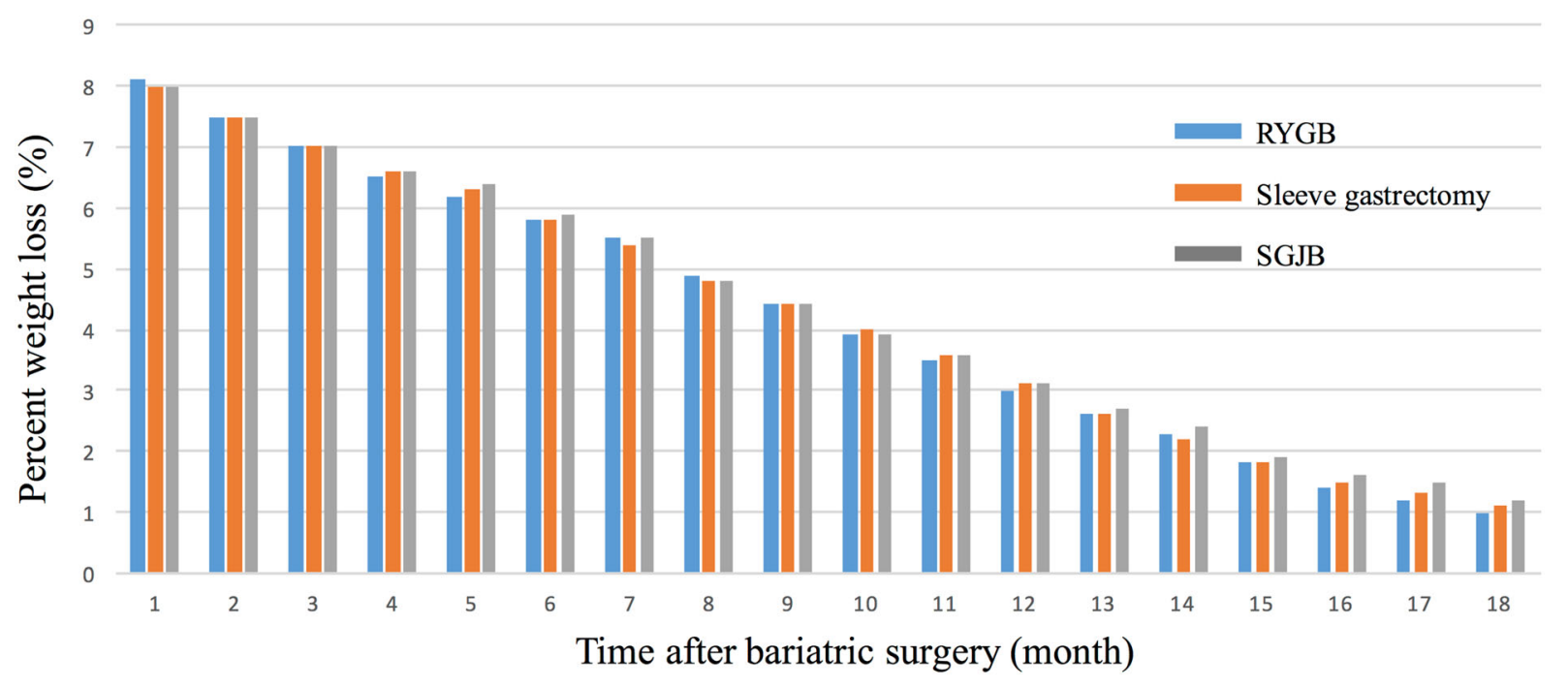

Fig. 2 Percentage weight loss every month after bariatric surgery

significant differences of the percentage weight loss after RYGB, sleeve gastrectomy, and SGJB.
As expected, the BMI declined each month after bariatric surgery, quickly in the first few months and then more gradually to stay steady 


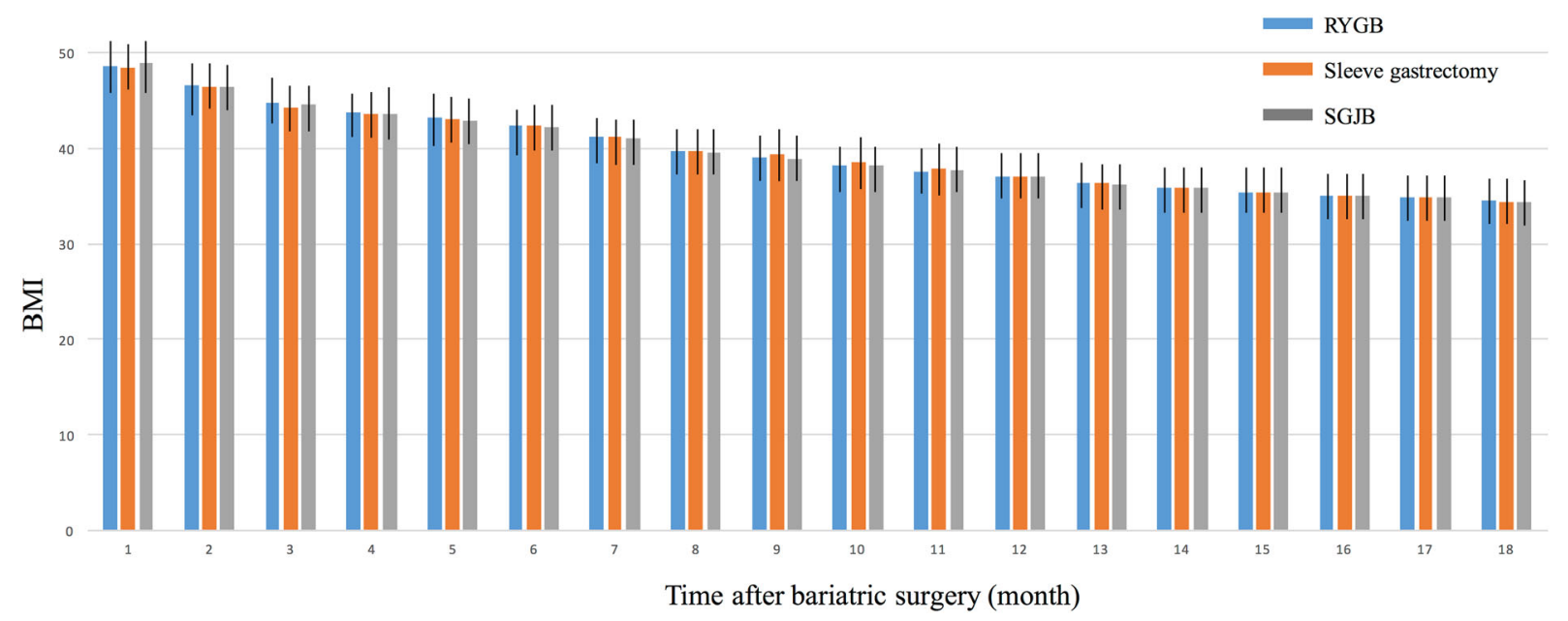

Fig. 3 BMI for every month after bariatric surgery

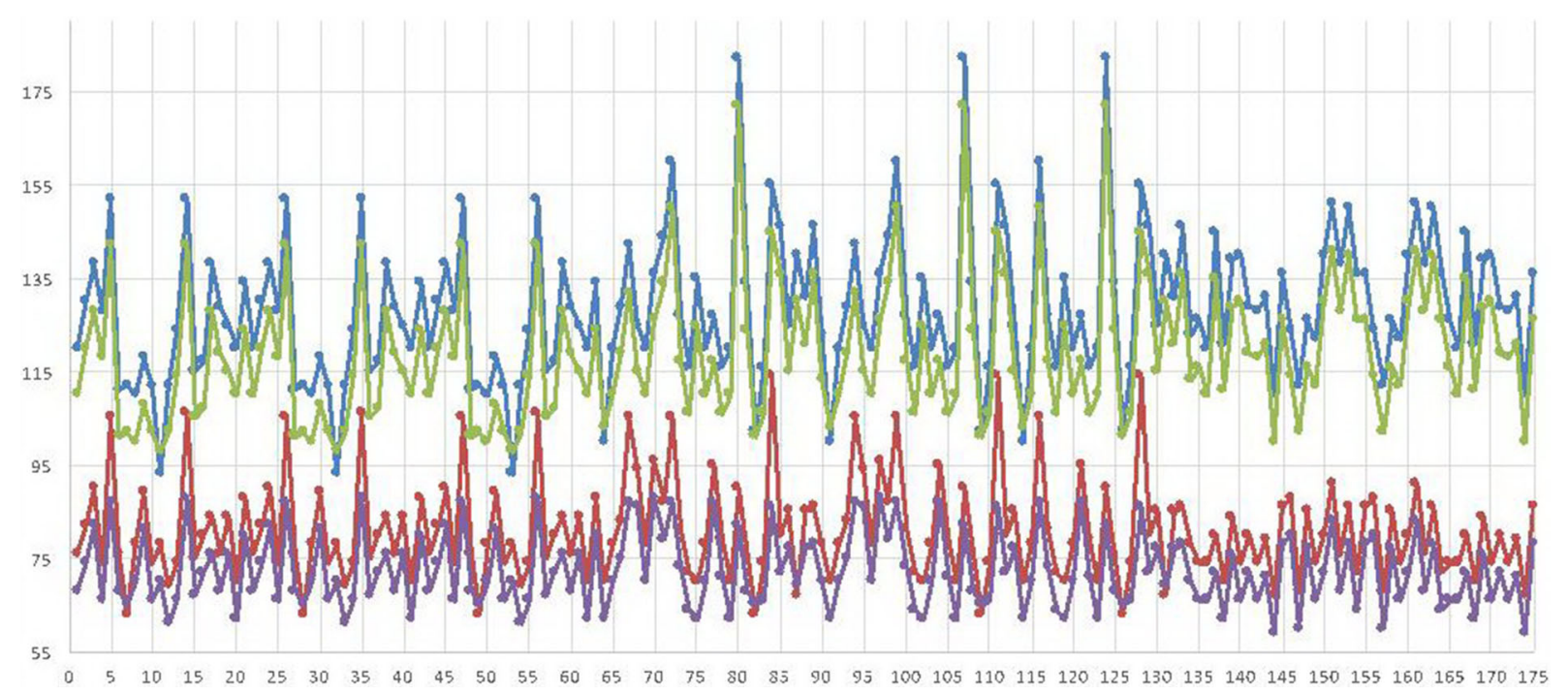

Fig. 4 Systolic and diastolic blood pressures for every patient before and after bariatric surgery. Note: Blue means the systolic pressure before bariatric surgery; red means the

(Fig. 3). There was also no significant difference of BMI variance among RYGB, sleeve gastrectomy, and SGJB.

Surprisingly, the systolic and diastolic blood pressures were much improved for every patient after bariatric surgery. As shown in Fig. 4, the systolic and diastolic blood pressures before bariatric surgery were more above the normal level for most obese patients; however, after bariatric surgery and regardless of the method diastolic pressure before bariatric surgery; green means the systolic pressure after bariatric surgery; and purple means the diastolic pressure after bariatric surgery

chosen, the systolic and diastolic blood pressures were nearly back to normal for most patients after an 18-month recovery. Moreover, other measurements, such as hyperlipidemia, hyperuricemia, fatty liver, obstructive sleep apnea syndrome, polycystic ovary syndrome and menstrual disorders for female obese patients, were improved to some degree. Patients after bariatric surgery, no matter which one kind of bariatric surgery, can keep their 
Table 2 Outcomes of weight loss based on the three kinds of bariatric surgery

\begin{tabular}{lllll}
\hline Variable (mean \pm SD) & RYGB & Sleeve gastrectomy & SGJB & $P$ value \\
\hline \%TWL & $29 \pm 10$ & $30 \pm 10$ & $30 \pm 10$ & 0.452 \\
\%EWL & $59 \pm 19$ & $58 \pm 20$ & $61 \pm 20$ & 0.393 \\
\%EBMIL & $54 \pm 12$ & $53 \pm 13$ & $54 \pm 13$ & 0.483 \\
$\Delta \mathrm{BMI}$ & $14 \pm 5$ & $13 \pm 5$ & $14 \pm 6$ & 0.491 \\
\hline
\end{tabular}

$P$ values indicate ANCOVA across all groups, controlling for follow-up time after surgery

$\% T W L$ percentage total weight loss, \%EWL percentage excess weight loss, \%EBMIL percentage body mass index loss, $B M I$ body mass index, $S D$ standard deviation

blood sugar levels stable and in the normal range under doctor's instruction and rational drug use. The results suggest that bariatric surgery can effectively improve the obesity-related complications once weight is steadily reduced.

From the statistics of patients undergoing RYGB, sleeve gastrectomy, and SGJB for about 18 months, we calculated the percentage total weight loss, excess weight loss, and BMI loss, and the overall BMI loss for every patient (Table 2). We found that there was no significant difference among the three types of bariatric surgery. It would cost 35,000-40,000 yuan for sleeve gastrectomy, about 45,000 yuan for SGJB, and about 45,000-50,000 yuan for RYGB. Under normal circumstances, it was usually 7-10 days of hospitalization after surgery, and there was no difference between the three kinds of surgery. To our surprise, all patients enlisted in our telephone interview followed the doctor's advice to maintain exercise and a diet for at least 1-2 years after surgery, while some patients returned to hospital for the second surgery or other ways to lose weight because of poor dietary habits or lack of exercise.

\section{DISCUSSION}

Patients admitted to our hospital for obesity were unable to rely on self-control of diet and exercise to keep fit and healthy. They were desperate to ask doctors for help and action was taken as soon as possible. On the basis of these conditions, we recommended patients undergo bariatric surgery and then remain on a diet. A very strict preoperative examination is required before surgery, including chest X-ray, electrocardiogram, abdominal ultrasound or CT to check whether patients can withstand surgery and anesthesia. Also, the level of aldosterone, estrogen, androgen, and pituitary MRI should be applied to exclude other diseases leading to obesity [15]. For example, pituitary adenoma, which could secrete growth hormone causing Cushing's syndrome, could also lead to obesity. In such cases bariatric surgery has no effect; only resection of pituitary adenoma is curative [16].

Laparoscopic sleeve gastrectomy is suitable for young patients with better islet function, mild diabetes, and insulin resistance [17]. If the patient has severe diabetes and islet dysfunction, RYGB surgery is recommended [18]. However, in terms of the specific surgical method, the doctor will make a detailed examination, assessment of the islet function and general condition of the patient, and then confirm a suitable surgical method for the patient. For sleeve surgery, ghrelin level dramatically dropped a short time after surgery and then remained at a lower level than that before surgery for almost all patients.

Weight loss surgery does not immediately make you thin. Loss of most weight happens in the first month; then less weight is lost than in the previous month. Surgery can only address $70 \%$ of extra body weight of a patient, rather than making the weight normal. A weight loss diet is very important after the operation [19]. Weight is easily gained with high-calorie food after surgery, so it is necessary to control the 
diet, change lifestyle, and combine anaerobic exercise and aerobic exercise.

Some patients were unable to stick to their diet and exercise during long-term recovery after their first surgery, and they had to return to our hospital for reoperation or hospitalization. We rechecked their body indexes and reevaluated whether they could tolerate another operation or other treatment. For example, three patients were rehospitalized. We initially made detailed arrangements for them to monitor their body weight change and other characteristics in the first 2 weeks. Two of the patients were kept under supervision, but the other patient required another operation.

This study was limited by the small population in 2 years, and use of more patients with longer follow-up information would have been more robust and precise.

\section{CONCLUSION}

This study compared three kinds of laparoscopic bariatric surgery and weight loss outcomes in individuals for 1-2 years after weight loss surgery. We found that bariatric surgery is the first choice, then long-term dietary control and exercise for weight loss, but it had nothing to do with the specific type of bariatric surgery.

\section{ACKNOWLEDGEMENTS}

We thank the participants of the study.

Funding. No funding or sponsorship was received for this study or publication of this article. The Rapid Service Fee was funded by the authors.

Authorship. All named authors meet the International Committee of Medical Journal Editors (ICMJE) criteria for authorship for this article, take responsibility for the integrity of the work as a whole, and have given their approval for this version to be published.

Compliance with Ethics Guidelines. All tests and individuals' personal health information related to human participants were performed in accordance with the ethical standards of the institutional and/or national research committee, and compliance with the basic principles of the Helsinki declaration of 1964 and its later amendments or comparable ethical standards.

Disclosures. Dan Luo, Qingling Yang, Li Zhou, Haibo Wang, Feng Li, Hailong Ge, He Huang, Jian Liu, and Yanjun Liu have nothing to disclose.

Data Availability. All data generated or analyzed during this study are included in this published article/as supplementary information files.

Open Access. This article is distributed under the terms of the Creative Commons Attribution-NonCommercial 4.0 International License (http://creativecommons.org/licenses/ by-nc/4.0/), which permits any noncommercial use, distribution, and reproduction in any medium, provided you give appropriate credit to the original author(s) and the source, provide a link to the Creative Commons license, and indicate if changes were made.

\section{REFERENCES}

1. Maciejewski ML, Arterburn DE, Van Scoyoc L, et al. Bariatric surgery and long-term durability of weight loss. JAMA Surg. 2016;151(11):1046-55.

2. Kwok CS, Pradhan A, Khan MA, et al. Bariatric surgery and its impact on cardiovascular disease and mortality: a systematic review and meta-analysis. Int J Cardiol. 2014;173(1):20-8.

3. Simonson DC, Halperin F, Foster K, Vernon A, Goldfine $\mathrm{AB}$. Clinical and patient-centered outcomes in obese type 2 diabetes patients 3 years after randomization to Roux-en-Y gastric bypass surgery versus intensive lifestyle management: the SLIMMT2D study. Diabetes Care. 2018;41(4):670-9.

4. Ahmed B, King WC, Gourash W, et al. Long-term weight change and health outcomes for sleeve gastrectomy (SG) and matched Roux-en-Y gastric bypass (RYGB) participants in the Longitudinal 
Assessment of Bariatric Surgery (LABS) study. Surgery. 2018;164(4):774-83.

5. Naitoh T, Kasama K, Seki Y, et al. Efficacy of sleeve gastrectomy with duodenal-jejunal bypass for the treatment of obese severe diabetes patients in Japan: a retrospective multicenter study. Obes Surg. 2018;28(2):497-505.

6. Ikramuddin S, Billington CJ, Lee WJ, et al. Roux-en$\mathrm{Y}$ gastric bypass for diabetes (the Diabetes Surgery Study): 2-year outcomes of a 5-year, randomised, controlled trial. Lancet Diabetes Endocrinol. 2015;3(6):413-22.

7. Purnell JQ, Johnson GS, Wahed AS, et al. Prospective evaluation of insulin and incretin dynamics in obese adults with and without diabetes for 2 years after Roux-en-Y gastric bypass. Diabetologia. 2018;61(5):1142-54.

8. Tassinari D, Berta RD, Nannipieri M, et al. Sleeve gastrectomy: correlation of long-term results with remnant morphology and eating disorders. Obes Surg. 2017;27(11):2845-54.

9. Ettleson MD, Lager CJ, Kraftson AT, et al. Roux-en-Y gastric bypass versus sleeve gastrectomy: risks and benefits. Minerva Chir. 2017;72(6):505-19.

10. Sepulveda M, Alamo M, Lynch R, et al. Comparison of long-term weight loss between sleeve gastrectomy and sleeve gastrectomy with jejunal bypass. Surg Obes Relat Dis. 2017;13(10):S58.

11. Sepúlveda M, Alamo M, Preiss $Y$, et al. Metabolic surgery comparing sleeve gastrectomy with jejunal bypass and Roux-en-Y gastric bypass in type 2 diabetic patients after 3 years. Obes Surg. 2018;28(11): 3466-73.
12. Watson KB, Frederick GM, Harris CD, et al. US adults' participation in specific activities: Behavioral Risk Factor Surveillance System-2011. J Phys Activ Health. 2015;12(6 Suppl 1):S3-10.

13. Stang A. Critical evaluation of the Newcastle-Ottawa scale for the assessment of the quality of nonrandomized studies in meta-analyses. Eur J Epidemiol. 2010;25(9):603-5.

14. DerSimonian R, Kacker R. Random-effects model for meta-analysis of clinical trials: an update. Contemp Clin Trials. 2007;28(2):105-14.

15. Taguchi M, Weiss AL. Examining pre-operative comorbidities and weight loss in adolescent bariatric surgery. J Adolesc Health. 2018;62(2):S124.

16. Atkins KM, Bussière M, Shih HA. Pituitary adenoma hypofractionated and stereotactic radiation therapy. Cham: Springer; 2018. p. 105-14.

17. Alaeddine MH, Shamseddine GA, Safadi BY. Laparoscopic sleeve gastrectomy operative dictations in general and vascular surgery. Cham: Springer; 2017. p. 131-3.

18. Camastra S, Astiarraga BD, Barbieri C, et al. Beneficial effects of RYGB on B-cell function and hepatic and peripheral insulin sensitivity are maintained seven years after surgery in both diabetic and nondiabetic subjects. Diabetes. 2018;67(Suppl. 1). https://doi.org/10.2337/db18-2089-P.

19. Barbieri P. Eat less or exercise more? Dieting efforts and price policies. SSRN. 2017. https://doi.org/10. 2139/ssrn.2689501. 